\title{
RECHERCHES SUR LES COCCIDIES INTESTINALES
}

\section{DES TRITONS}

\author{
Par G, LA VIER
}

Il y a déjà bien longtemps que la coccidiose intestinale des tritons a été signalée ; en fait, c'est une des plus anciennement connues. Dès 1881, Aimé Schneider décrivait en détail, sous le nom d'Orthospora propria, une Coccidie dont il avait rencontré les oocystes dans le tube digestif de Triton cristatus et Triton vulgaris des environs de Poitiers. Plus tard, en 1887, il abandonna le genre Orthospora qu'il avait créé pour cette unique espèce et la fit rentrer dans le genre Coccidium dont elle avait le mode de segmentation oocystique ; il signala en même temps, dans la coccidie du triton, deux « faciès », l'un Coccidium proprium dont l'oocyste est cylindroïde et l'autre Coccidium sphericum avec oocyste régulièrement sphérique; notons tout de suite que, dans ce mémoire de 1887 , il il ne donne pas de diagnose de cette nouvelle forme, mais de bonnes figures et que si, dans le texte, il emploie pour elle le terme de " faciès », dans la légende de sa planche, il utilise celui d" " espèce ».

Mais, pour A. Labbé (1893), les deux Coccidium de Schneider " ne sont ni deux espèces, ni mème deux variétés différentes ;... les Coccidium peuvent être soumis à tous les caprices du développement, relativement à la taille et à la forme de la capsule et, par conséquent, il ne faut pas tenir compte de ces deux caractères lorsqu'on fait une spécification. Nous énonçons donc », conclut-il, " cette coccidie: Coccidium proprium. "Si extraordinaires que puissent nous paraitre aujourd'hui ces prémisses, la conclusion que Labbé a maintenue en 1896 et 1899 semble avoir été généralement acceptée jusqu'à maintenant.

D'autre part, on étudiait le cycle endogène. Ce qu'en avait observé Schneider dans les cellules épithéliales se rapportait seulement aux macrogamètes. Steinhaus (1891) décrit, sous le nom de Cytophagus tritonis, des stades schizogoniques d'une coccidie dont il ne voit pas de forme kystique. En 1896, A. Labbé range les stades intracellulaires déjà connus de Coccidium proprium dans son fameux genre Pfeifferia que déjà rien ne pouvait justifier, comme le montrait aus-

Annales de Parasitolosie, T. XIV, N². - $1^{\text {er }}$ mars 1936 , p. 150-163. 
sitòt P.-L. Simond entre auţres ; ce dernier auteur (1897) réalisait en outre des infections expérimentales de tritons ; peu après, Siedlecki (1898) faisait connaitre la reproduction sexuée et le début de la sporulation.

Depuis, la coccidiose intestinale des tritons a dû être observée par un nombre considérable d'observateurs, étant donné sa grande fréquence et la facilité avec laquelle on peut se procurer le matériel d'étude, mais elle n'a pas, à ma connaissance du moins, donné lieu à des recherches nouvelles. Tous les traités, quelle qu'en soit la langue, admettent qu'elle est due à l'espèce décrite par Schneider et devenue, après de longues controverses de nomenclature générique qu'il est inutile de rappeler, Eimeria propria (A. Schneider, 1881).

Au cours d'un séjour, en mai 1934, à la Station aquicole Grimaldi à Saint-Jean-de-Losne (Côte-d'Or), j'ai eu l'occasion de rencontrer un Triton vulgaris dont l'intestin contenait de très abondants oocystes coccidiens. Ayant eu la curiosité de me référer à la description originale d'A. Schneider, j'eus la surprise de constater qu'elle était, sur plusieurs points, en désaccord avec ce que je pouvais observer. En particulier, je recherchai en vain, sur la paroi de l'oocyste, une particularité que cet auteur donne cependant comme constante : " la zone équatoriale présente, sur la coupe optique de la paroi, une série de raies transversales... qui me semblent être l'expression de très fins canalicules poreux. 》D'ailleurs, dans la description très détaillée de Schneider, reviennent fréquemment les termes : « tantòt..., tantòt... ». C'est ainsi que le contenu de l'oocyste est tantôt « pur, à fines granulations moléculaires », tantôt « renferme un nombre variable, souvent considérable, de grosses gouttelettes de graisse ». D'autres alternatives concernent la formation des spores ou encore le reliquat cystique qui est, tantôt « sphérique ou subsphérique, granuleux, en partie vacuolarisé, tantôt un amas de grosses gouttelettes de graisse ». Or, outre l'absence constante des canalicules poreux, les oocystes que j'avais sous les yeux présentaient une grande uniformité d'aspect tant avant qu'après la segmentation; aucun, en particulier, ne montrait ces " gouttelettes de graisse », signalées comme si fréquentes par Schneider. L'existence de ces dernières, ainsi que celle des canalicules, affirmée par un tel observateur, ne pouvait cependant être mise en doute. C'est ainsi que je fus amené à reprendre l'étude complète de la coccidiose intestinale des tritons.

Grâce à l'amabilité du Professeur P. Paris, de Dijon, je pus avoir à ma disposition un grand nombro: de tritons appartenant aux espèces $T$. vulgaris, $T$. palmatus, $T$. slpestris et $T$. cristatus, prove- 
nant tous de Saint-Jean-de-Losne (Còte-d'Or). L'étude seule des oocystes suffit à me convaincre que l'on rencontrait chez eux; non pas une seule, mais bien quatre espèces différentes d'Eimeria, dont Schneider avait d'ailleurs bien vu les formes, mais que, à l'exception de $E$. spherica, il avait confondues. Sans doute, avait-il eu affaire à des infections mixtes, alors que j'avais eu, dès le début, la chance qui se renouvela par la suite, de tomber sur une infection pure. J'eus ainsi, à diverses reprises, un matériel ne prêtant pas à l'erreur pour étudier la forme et la segmentation des oocystes et les stades intraépithéliaux.

Comme les caractères tirés des oocystes sont à la fois les plus nets et les plus faciles à observer, c'est par eux que je commencerai et sur eux que je baserai la distinction des espèces.

\section{Formes oocystiques}

Avant de passer à leur examen systématique, je voudrais tout d'abord rapporter quelques observations d'ordre général.

Quand on examine le contenu intestinal d'un triton que l'on vient de sacrifier, il est très fréquent, presque constant même, d'y rencontrer une forte proportion d'oocystes à des stades divers de la segmentation, et même parvenus à maturité complète. Cela pourrait peut-être s'expliquer par la grande lenteur du transit intestinal chez ces animaux qui permet à la sporulation de s'achever avant l'expulsion dans le milieu extérieur. Cette lenteur de transit est attestée par la présence, après plus de huit jours de captivité, d'éléments de nature animale ou végétale provenant du plancton du lieu de pêche. Dans un seul cas (infection pure par E. propria), il n'y avait pas, à l'ouverture de l'intestin, d'oocystes segmentés; ils l'étaient tous entièrement 24 heures après ; mais, comme dans presque tous les individus, le cytoplasme, au moment de la mise en culture, était déjà contracté en śphère, ce laps de temps doit être considéré comme inférieur à la réalité ; aussi ne puis-je le donner qu'à titre indicatif. Il n'y aura donc pas lieu de s'étonner si je ne fournis, par la suite, aucun renseignement sur la vitesse de segmentation dans les quatre espèces différentes ; j'ai seulement l'impression qu'à cet égard, elles doivent se comporter sensiblement de mème façon.

J'ai pu encore constater un phénomène curieux : la taille des oocystes a tendance à augmenter avec le temps. Je l'ai très nettement observé dans les cultures faites en acide chromique à 5 p. 1000; mais cela n'est en rien dû à ce liquide, car le mème phénomène se passe dans l'intestin mème du triton où les oocystes déjà seg- 
mentés sont nettement plus grands et le sont d'autant plus que la segmentation est plus avancée. Cette augmentation progressive de la taille est importante à connaitre pour l'exactitude des dimensions, car elle peut dépasser $5 \mu$; aussi, pour éliminer ce facteur, ne donnerai-je plus loin, comme dimensions, que celles des oocystes non segmentés.

Un autre point intéressant, c'est que ces formes coccidiennes différentes se rencontrent chez les divers tritons sans égard pour leur espèce ; c'est là un fait assez rare dans le genre Eimeria pour qu'il soit digne d'être noté ; dans la plupart des cas, en effet, ces coccidies sont strictement spécifiques même vis-à-vis d'hôtes très voisins.

Enfin, parmi les tritons que j'ai examinés, certains ont été tenus en eaptivité plusieurs semaines dans une promiscuité étroite qui aurait dû favoriser les échanges d'espèces parasitaires. Cependant, j'ai encore rencontré, à plusieurs reprises, des infections pures. Cela peut, peut-être, s'expliquer par une immunité de groupe ayant empêché les surinfections.

Voyons maintenant successivement les quatre espèces (fig. 1).

I. Eimeria propria (A. Schneider, 1881) emend. (fig. 1, A ; fig. 2). - L'oocyste est cylindroïde, comme l'a décrit Schneider, allongé avec extrémités symétriquement arrondies, mesurant habituellement en longueur de 38 à $41 \mu$ (minimum : 36 ; maximum : 43), sur une largeur de 22 à $24 \mu$ (minimum : 20 ; maximum : 27); l'indice morphologique (quotient de la largeur par la longueur) est en moyenne de 0,58 à 0,60 (minimum : 0,51 ; maximum : 0,67 ).

La paroi est double, présentant une épaisseur totale de $0 \mu, 8$ environ et partout identique, dépourvue de micropyle et d'ornementation. Sa couche interne est plus mince que l'externe. Le contenu avant segmentation emplit l'oocyste ; il renferme de petites granulations réfringentes. Au début de la segmentation, il se rassemble en une sphère qui n'est pas médiane, mais plus rapprochée d'un pôle que de l'autre; pendant quelque temps d'ailleurs, la sphère reste attachée au pôle proximal par une traìnée granuleuse que Schneider avait nommée filament suspenseur. Pour lui, elle part d'un « stigma », petite saillie légère que la membrane interne fait dans la cavité de l'oocyste. Je n'ai jamais pu, en réalité, observer ce stigma ; ce que j'ai vu fréquemment, et ce qui, sans doute, a provoqué cette interprétation de Schneider, c'est un petit granule réfringent analogue à ceux que l'on peut voir sur le « filament suspenseur », et qui, s'il est appliqué contre la membrane interne, n'en fait néanmoins pas partie et souvent d'ailleurs en est détaché 
et nettement distant. Quant au filament suspenseur (fig. 2, $a-c$ ), cette très curieuse formation, qui évoque l'idée d'un chalaze d'œuf de poule, me paraît constituée par une traînée cytoplasmique hyaline maintenant en cohésion quelques granules réfringents; sa durée
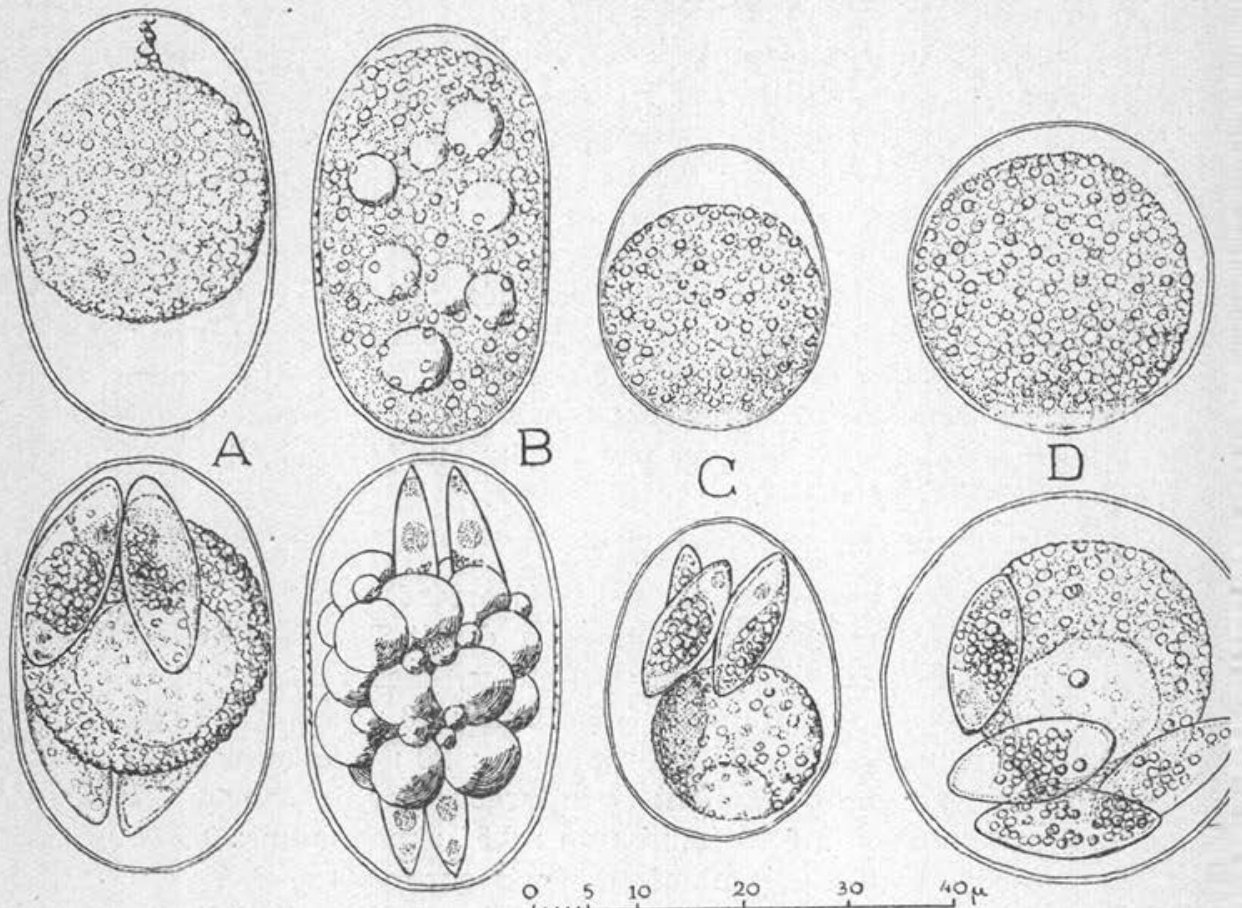

Fig. 1. - Oocystes des quatre espèces d'Eimeria parasitant l'intestin des tritons indigènes. (Dans cette figure, comme dans les suivantes, la paroi qui comprend en réalité deux couches différentes a été représentée comme simple) : A, Eimeria propria (A. Schneider) emend.; en haut, oocyste tout au début de la segmentation : condensation du cytoplasme, existence du \& filament suspenseur » de Schneider; en bas : segmentation terminée. B, Eimeria canaliculata $\mathrm{n}$. sp. ; en haut : oocyste avant segmentation ; en bas : segmentation terminée. C, Eimeria tertia n. sp. ; en haut : oocyste au début de la condensation cytoplasmique ; en bas : segmentation terminée. D, Eimeria spherica (A. Schneider); en haut : oocyste non segmenté ; en bas : segmentation terminée.

est d'ailleurs limitée et bientòt elle disparait, libérant les granules dont le nombre diminue vite ; ceux-ci s'évanouissent à leur tour, sans doute résorbés par métabolisme et avant que, dans la sphère, l'ébauche des spores n'apparaisse, rien ne reste du tractus qui, primitivement, la reliait à la paroi. 
Dans cette sphère granuleuse, se différencient les quatre spores et le reliquat cystal. La segmentation en cours donne à l'ensemble un aspect polyédrique (fig. $2, d$ ) ; quand elle est achevée, les quatre masses sporales détachées présentent encore un aspect pyramidal
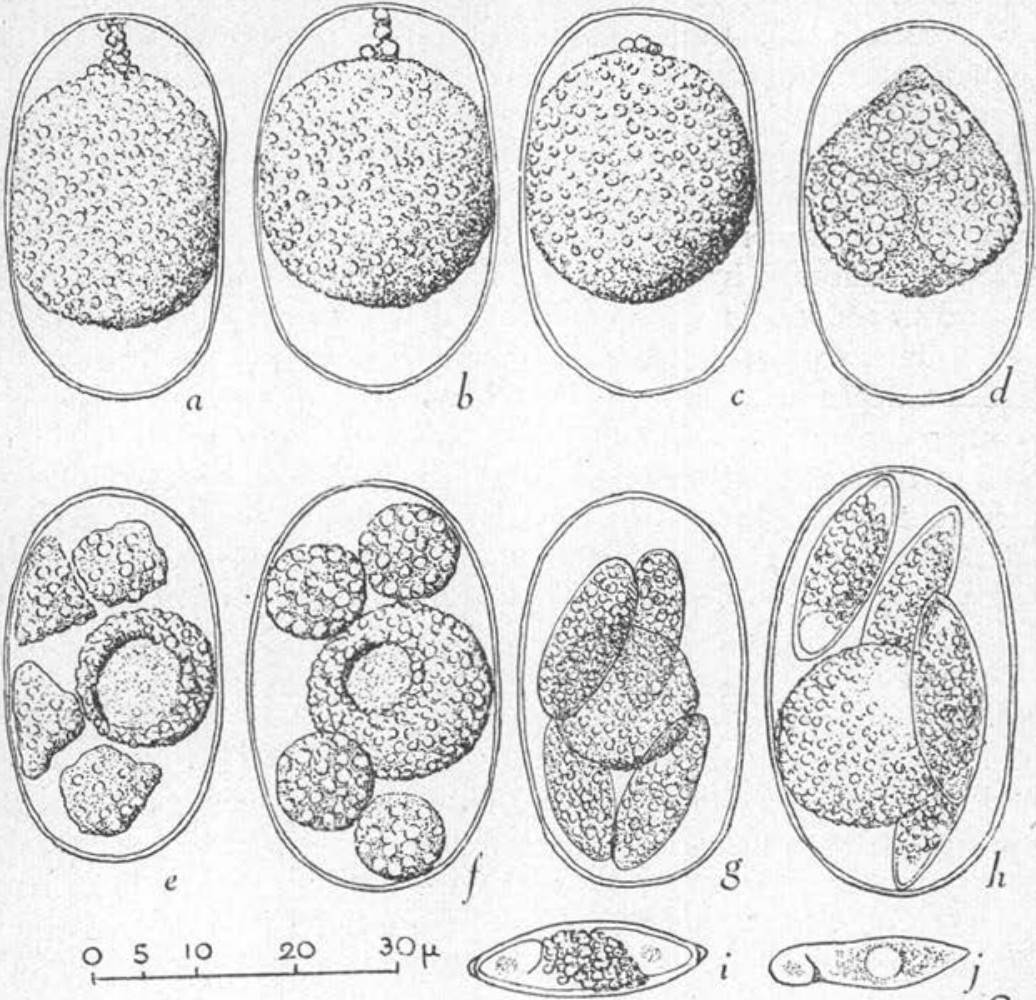

FıG. 2. - Eimeria propria (A. Schneider). $a-c$, résorption du « filament suspenseur $\gg ; d$, début de la segmentation ; $e$, individualisation des quattre spores et du reliquat ; $f$, arrondissement des spores; $g$, allongement des spores ; $h$, formation des sporozoïtes ; $i$, une spore à maturité ; $j$, un sporozoïte extrait par pression.

(fig. $2, e$ ) qu'elles perdent vite pour s'arrondir en sphères de 8 à $10 \mu$ environ de diamètre (fig. $2, f$ ) ; leur taille augmente d'ailleurs et elles se déforment pour s'allonger en ellipsoïdes (fig. $2, g$ ); les deux extrémités, symétriques, d'abord très arrondies deviennent plus effilées (fig. $2, h$ ) mais en restant toujours mousses. A la 
maturité, ces spores (fig. $2, i$ ) mesurent de 18 à $22 \mu$ de longueur sur 7 à 8 de largeur ; elles contiennent alors deux sporozoïtes placés têtebêche et un reliquat sporal granuleux ; celui-ci forme un amas ellipsoïdal d'environ $10 \mu$ sur 7 ; il ávait déjà attiré l'attention de Schneider qui l'avait appelé « le bouclier »; il est, en effet, toujours bien ramassé, mais parfois quelques granules le prolongent sur les côtés, s’insinuant entre la paroi et les sporozoïtes.

Quant au reliquat cystal, il forme une sphère creuse mesurant au début de 11 à $12 \mu$ de diamètre, mais qui augmente peu à peu de taille tout en diminuant de densité ; cette sphère est creuse, en forme de gastrula, présentant un orifice circulaire facile à observer. Les granules qui la composent sont maintenus en cohésion par une sorte de membrane hyaline qui les recouvre et qui se voit bien dans les oocystes dont la segmentation est terminée depuis plusieurs jours ; dans ce cas, en effet, le diamètre du reliquat peut atteindre et même dépasser $22 \mu$, les granulations sont presque toutes isolées les unes des autres et leur nombre est sensiblement diminué. Il est difficile, en constatant cette disparition progressive des granulations réfringentes du reliquat, de ne pas leur attribuer un rôle de réserves nutritives. Parfois, on peut voir, à l'intérieur de la sphère, un granule plus gros que les autres ; mais ce phénomène est beaucoup moins constant ici que chez E. spherica.

Par pression, on peut libérer les sporozoïtes mesurant de 15 à $18 \mu$ de longueur sur 2,5 de largeur et présentant l'aspect habituel des sporozoïtes coccidiens (fig. $2, j$ ) ; cette manœuvre déforme, mais ne dissocie pas le reliquat cystal à cause de la membrane qui le limite, mais elle éparpille les granules des reliquats sporaux.

Cette espèce coccidienne est commune ; je l'ai rencontrée à l'état pur chez un Triton vulgaris et, en association à diverses reprises chez $T$. cristatus et $T$. alpestris. Elle présente sensiblement la mème fréquence que l'espèce suivante avec laquelle Schneider l'avait confondue. L'oocyste en est cependant facile à différencier en se basant sur les trois caractères suivants : paroi lisse ; contenu finement granuleux ; segmentation donnant un gros reliquat sphérique.

C'est cette espèce qui, à mon avis, a droit au nom spécifique créé par Schneider, car c'est à elle que s'appliquent la majorité des caractères donnés par cet auteur, en tous cas ceux que dans son texte il a énoncé en premier lieu.

II. Eimeria canaliculata n. sp. (fig. 1, B ; fig. 3). - Les oocystes de cette espèce ont sensiblement la même forme et les mèmes dimensions que ceux de l'espèce précédente : longueur, de 39 à $40 \mu$ (minimum : 36 ; maximum : 42) ; largeur : de 23 à $25 \mu$ (minimum : 
20 ; maximum : 27) ; indice morphologique moyen : 0,58 à 0,60 (maximum : 0,67 ; minimum : 0,53).

Mais ils présentent deux différences constantes et faciles à observer, portant l'une sur la paroi, l'autre sur le contenu.

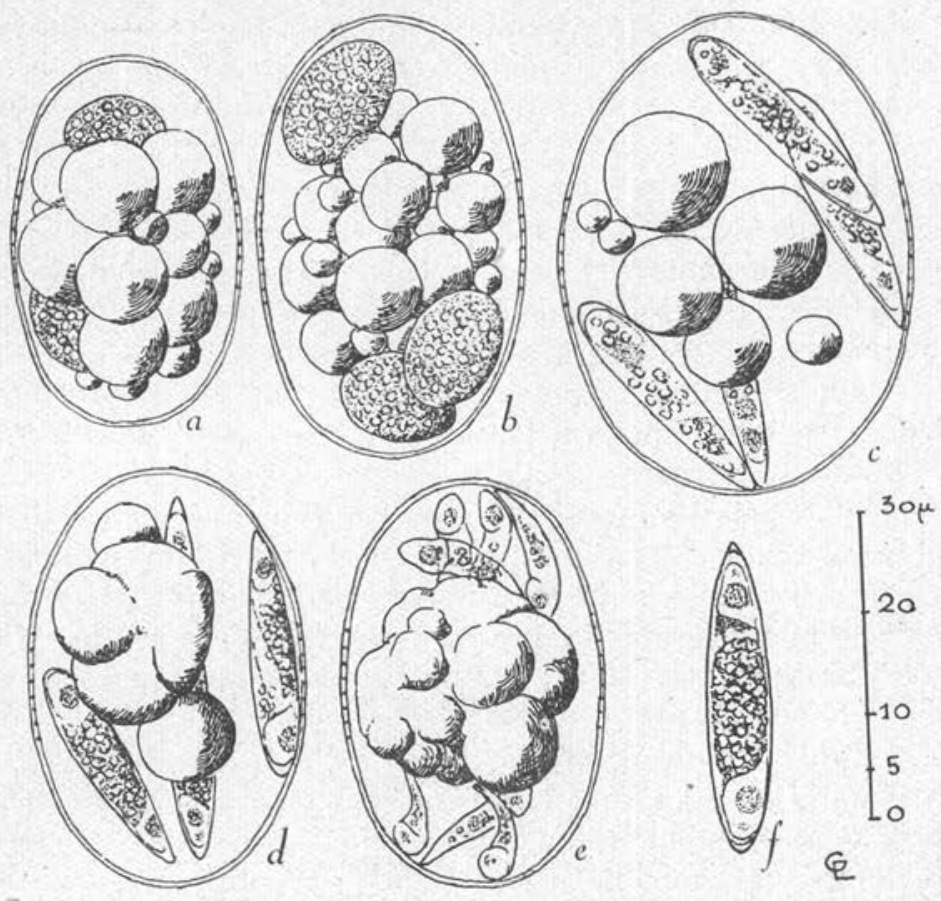

Fig. 3. - Eimeria canaliculata n. sp. $a$, apparition des masses sporales ; $b$, allongement de ces masses ; $c$, oocyste plusieurs jours après la fin de la segmentation; le reliquat oocystique est réduit à quelques gros globules; les reliquats sporaux ont également beaucoup diminué d'importance ; $d$, autre oocyste plusieurs jours après la fin de la segmentation, les gros granules devenus coalescents forment une masse unique multilobulée : e, anomalie de segmentation : absence d'enveloppes sporales, les sporozoïtes sont nus et libres dans l'oocyste; les globules du reliquat sont coalescents ; $f$, spore à maturité.

La paroi est plus épaisse, mesurant environ $1 \mu$; elle est double et sa couche interne présente une particularité remarquable : dans la zone équatoriale, elle est forée de fins canalicules radiants. Schneider avait bien relevé ce détail, unique d'ailleurs dans la structure des Coccidies, mais l'avait, à tort, généralisé. Ces curieux canalicules ne m'ont pas paru, comme à lui, traverser entièrement la paroi, ce qui serait d'ailleurs fort dangereux pour la vie du contenu; je ne crois 
même pas qu'ils atteignent la couche externe et j'ai plutôt l'impression qu'ils se terminent borgnes dans la couche interne. Je n'ai jamais pu les observer que de profil ; mais, dans cette position, ils sont toujours visibles ; on peut donc en conclure qu'ils sont répartis sur une bande équatoriale qui mesure de 12 à $13 \mu$ de diamètre ; leur nombre n'est pas quelconque et, dans les bonnes conditions de visibilité, j'en ai toujours compté 8 ou 9 ; cette possibilité de deux nombres voisins me ferait volontiers admettre qu'ils sont disposés en quinconce.

Le cytoplasme de l'oocyste contient les petites granulations habituelles chez les Coccidies ; mais parmi elles, on observe constamment de gros granules sphériques très réfringents dont la taille peut atteindre jusqu'à 7-8 $\mu$ de diamètre (fig. $1, \mathrm{~B}$ ).

La segmentation va, par la suite, accentuer encore les différences. A son début se produit, comme c'est la règle, une condensation cytoplasmique ; elle se fait, sans laisser ce tractus que Schneider appelait filament suspenseur, et elle rassemble les grosses granulations qui étaient jusques-là éparses et dont l'agglomération va constituer d'emblée le reliquat kystique tandis que la partie finement granuleuse du cytoplasme donnera naissance aux spores. La formation de celles-ci n'est pas facile à voir, masquée qu'elle est par l'amas des gros granules (fig. $3, a$ ) ; dès qu'elles sont observables, elles se présentent sous l'aspect de masses sphéroïdales de $10 \mu$ environ de diamètre qui s'allongent bientôt (fig. $3, b$ ).

A la maturité, elles sont très élancées, mesurant habituellement de 25 à $30 \mu$ de longueur sur 6 de largeur ; une seule extrémité est aiguë, l'autre est arrondie (fig. $3, f$ ). Elles contiennent deux sporozoïtes placés tête-bêche et un reliquat sporal ovalaire de petites granulations, qui n'est pas exactement médian, mais un peu plus rapproché de l'extrémité arrondie que de celle qui pointe. On a alors un aspect très caractéristique de l'oocyste donné par l'amas de gros granules réfringents agglomérés d'où sortent les spores comme les épingles d'une pelote, avec leur extrémité aiguë dirigée vers la périphérie (fig. 1, B).

Autant què la paroi, le reliquat cystal est caractéristique de cette espèce ; les oocystes de coccidies présentent toujours d'abondantes granulations réfringentes, mais toutes de petit diamètre et d'aspect similaire, quelle qu'en doive être la destinée au cours de la segmentation. Avec Eimeria canaliculata, nous voyons, de façon frappante, que le cytoplasme avant la segmentation contient deux sortes d'inclusions, les unes qui sont destinées à faire partie des spores, les autres qui seront d'emblée mises à l'écart. Schneider considérait les 
gros granules comme des « gouttelettes huileuses »; c'était d'ailleurs là certainement un simple diagnostic d'impression, car il ne parle pas, dans son texte, de réactions microchimiques. Bien que je n'aie pas non plus pratiqué ces dernières (rendues d'ailleurs très difficiles par l'imperméabilité de la paroi), je puis affirmer qu'il ne s'agit pas de graisses ; en effet, tassées les unes contre les autres, comme elles le sont dans le reliquat cystal, des gouttelettes lipoïdiques fusionneraient en une goutte unique, alors qu'en réalité les gros granules conservent généralement leur individualité. Le reliquat d'ailleurs évolue avec le temps : progressivement, le nombre des globules diminue (fig. $3, c$ ) ; parfois, mais rarement, au cours de cette résorption, ils deviennent coalescents, mais leur ensemble forme alors, non pas une sphère unique, mais une masse à surface lobulée (fig. $3, d, e$ ). Tout cela donne à penser qu'il s'agit vraisemblablement de plastes vitellins qui sont utilisés pour la nutrition des spores.

J'ai eu l'occasion de rencontrer dans cette espèce un oocyste qui avait présenté une intéressante anomalie de sporulation (fig. 3,e) : l'absence d'enveloppes sporales ; les huit sporozoïtes qui étaient parfaitement normaux d'aspect, étaient libres dans l'oocyste ; le reliquat cystal présentait ses caractères habituels ; il n'y avait pas trace des reliquats sporaux. On sait que Laveran et Mesnil ont décrit, en 1902, une coccidie intestinale de Rana esculenta, pour laquelle ils ont créé le genre Paracoccidium, précisément basé sur la disparition de l'enveloppe sporale libérant les huit sporozoïtes. Mais, comme le fait remarquer Wenyon, en faisant tomber en synonymie le nom générique Paracoccidium avec celui d'Eimeria, le phénomène peut s'observer avec d'autres espèces appartenant indubitablement au genre Eimeria, E. falciformis, par exemple ; Wasielewski (1904) l'avait vu chez E. stiedx; E. canaliculata vient en fournir une preuve nouvelle.

Cette espèce nettement caractérisée est commune; elle se rencontre au moins aussi fréquemment, sinon plus, que la précédente ; je l'ai observée à l'état pur chez deux $T$. cristatus et, en association avec les autres espèces coccidiennes, à diverses reprises, chez plusieurs $T$. palmatus, $T$. vulgaris, $T$. alpestris et $T$. cristatus.

III. Eimeria tertia n. sp. (fig. 1, C ; fig. $4, a, d)$. - Schneider avait remarqué l'existence d'oocystes à contour ovalaire ; il les attribuait à un élargissement individuel : « quand le diamètre transverse, ditil, est plus considérable, la psorospermie devient nettement ovalaire »; sa figure 8 représente un tel oocyste.

Cette explication ne saurait, en réalité, être tenue pour suffi- 
sante ; en effet, l'élargissement du diamètre transversal (qui, d'ailleurs, se produit, comme nous l'avons vu dans les cultures) ne rend pas le contour ovalaire, mais le laisse elliptique ; les deux extrémités de l'oocyste restent toujours symétriques par rapport au plan
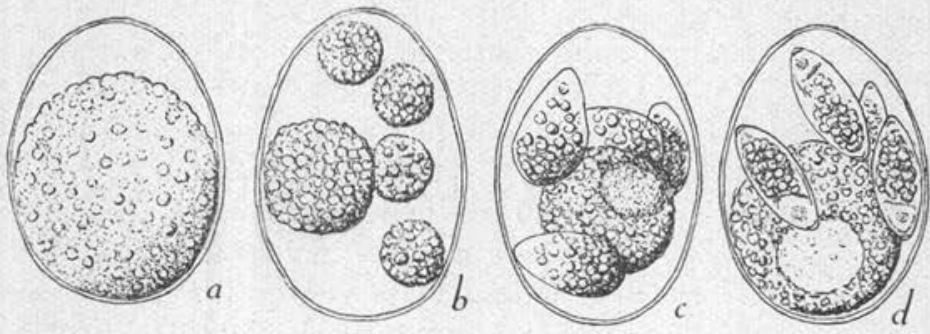

:
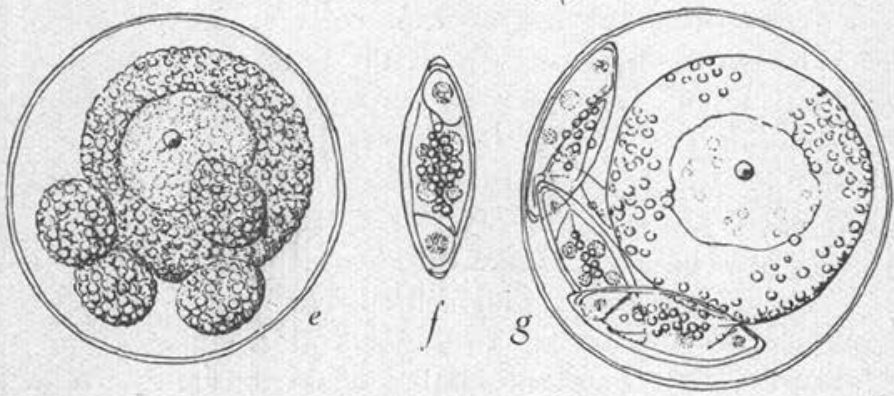

$\varepsilon$

Fig. 4. - a-d, Eimeria tertia n. sp., segmentation de l'oocyste ; e-g, Eimeria spherica (A. Schneider); $e$, individualisation des masses sporales et du reliquat ; $f$, spore à maturité ; $g$, oocyste plusieurs jours après la fin de la segmentation : les granulations du reliquat oocystique se sont raréfiées, le gros granule central est bien visible ainsi que la membrane ; les granulations sporales ont également diminué en quantité.

équatorial, tandis qu'avec des oocystes vraiment ovalaires, les deux extrémités ont des rayons de courbure nettement différents.

J'ai rencontré à deux reprises des oocystes ovalaires et de taille sensiblement inférieure à celle des espèces précédentes. Ils mesurent en effet en moyenne, $26 \mu$ (min. : 22 ; max. : 33 ) de longueur sur $21 \mu$ (min. : 18 ; max. : 25) de largeur ; leur indice morphologique varie de 0,70 à 0,88 ; il s'agit done d'une forme bien plus trapue que celles que nous venons de voir et, je le répète, ovalaire au sens exact du mot, au lieu d'être elliptique. 
Leur paroi est également plus mince, mesurant environ $0 \mu, 6$ d'épaisseur ; elle est double, la couche interne étant dépourvue d'ornementation. Le contenu cytoplasmique est finement granuleux.

La segmentation se fait suivant le même type que chez Eimeria propria (fig. $4, a-d$ ) ; elle donne un reliquat en sphère creuse mesurant de 12 à $17 \mu$ de diamètre et quatre spores ; celles-ci se terminent symétriquement à chaque extrémité par une pointe très arrondie ; elles mesurent de 12 à $15 \mu$ de longueur sur 6 à 7 de largeur. Elles contiennent deux sporozoïtes placés tête-bêche et un reliquat sporal granuleux rassemblé en un amas elliptique qui n'est pas exactement médian, mais plus rapproché d'une extrémité.

Je n'ai pas rencontré, à l'état pur, l'espèce coccidienne correspondant à cette forme oocystique; dans un cas ( $T$. alpestris), les kystes ovalaires étaient rares et associés à ceux d'Eimeria propria (abondants) et d'E. canaliculata (assez rares) ; dans le second cas, ils étaient un peu plus abondants et associés à $E$. canaliculata seule, et cela permet de trancher nettement la question de validité de l'espèce Eimeria tertia.

Elle présente en effet assez de caractères communs avec E. propria pour qu'on puisse se demander légitimement si, comme le eroyait Schneider, il ne s'agirait pas seulement d'individus particulièrement petits et trapus de cette espèce. Mais d'un côté, les infections pures à $E$. propria ne m'ont pas montré d'individus analogues ni comme forme, ni comme taille; de l'autre, la présence d'E. tertia en l'absence d' $E$. propria, montre bien l'individualitẻ de cette espèce.

IV. Eimeria spherica (A. Schneider, 1887) (fig. 1, D ; fig. 4,e-g). Cette espèce qu'avait décrite Schneider sous le nom de Coccidium sphericum et que Labbé a fait tomber en synonymie sans raisons, en 1893 , est parfaitement valable. J'ai pu en rencontrer une infection rigoureusement pure chez un Triton alpestris. Elle paraît toutefois être assez rare.

L'oocyste est régulièrement sphérique, le diamètre variant de 22 à $38 \mu$; chez les individus les plus nombreux, il est de $35 \mu$ environ. La paroi présente la même épaisseur que chez E. propria ; elle est double et sans ornementation ; le contenu est finement granuleux.

La segmentation se fait comme chez Eimeria propria (fig. 4,e) ; elle aboutit à quatre spores symétriques à extrémités mousses qui mesurent de 12 à $15 \mu$ de longueur sur 6 à 7 de largeur (fig. $4, f$ ). Elles contiennent deux sporozoïtes placés tête-bêche et un reliquat de quelques granules massés généralement au centre, mais avec une 
tendance à la dispersion. Le reliquat cystal est en sphère creuse ; le diamètre de celle-ci augmente d'ailleurs avec le temps jusqu'à atteindre et même dépasser $25 \mu$. Dans ces grands reliquats, comme dans ceux d'E. propria, les granules sont raréfiés et la membrane d'enveloppe est rendue ainsi bien visible (fig. $4, g$ ). On peut noter aussi, avec la plus grande facilité, la présence d'un granule réfringent central ou subcentral, dans la cavité de la sphère ; il est isolé et mesure de 2 à $3 \mu$ de diamètre ; il est donc nettement plus gros que les granulations ordinaires du reliquat. Ce granule est bien plus aisément observable chez E. spherica que chez E. propria. A. Schneider avait bien noté ce détail : «On distingue, dit-il, souvent, au centre de la volumineuse vacuole... un petit globule qui simule un nucléole dans un nucleus. 》 Je ne puis actuellement donner aucune interprétation de cette curieuse formation.

$$
* *
$$

Ainsi, nos tritons indigènes présentent quatre espèces différentes de Coccidies intestinales du genre Eimeria, que leur forme oocystique permet de grouper en deux catégories. La première comprend uniquement Eimeria canaliculata, que sa paroi canaliculée et ses gros globules vitellins écartent nettement des autres espèces connues du genre et qui constitue, par suite, une forme des plus intéressantes. La deuxième catégorie a pour type Eimeria propria et est caractérisée par la formation d'un reliquat bien organisé, en sphère creuse, pourvu d'une membrane et comportant souvent, peut-être même toujours, un granule réfringent central de nature énigmatique. Des reliquats analogues ont été décrits chez d'autres Eimeria que celles des tritons; par exemple, chez E. prevoti (Laveran et Mesnil, 1902), de Rana esculenta et chez E. delagei (Labbé, 1893), parasite du Chélonien, Cistudo europæa. Il y a donc là un caractère qui, étant donné la nature des hôtes, semble bien correspondre à des affinités précises.

\section{BiBLIOGRAPHIE}

LaBbÉ (A.). - Coccidium Delagei, coccidie nouvelle parasite des tortues d'eau douce. Arch. Zool. exp. et gén., série 3, I, 1893, p. 267.

- Recherches zoologiques, cytologiques et biologiques sur les Coccidies. Ibid., série 3, IV, 1896 , p. 517.

- Sporozoa. Das Tierreich., Berlin, 1899.

Laveran (A.) et Mesnil (F.). - Sur deux coccidies intestinales de la Rana esculenta. C.R. Soc. de Biol., LIV, 1902, p. 857. 
Schneider (A.). - Sur les psorospermies oviformes ou Coccidies. Arch. Zool. exp. et gén., IX, 1881, p. 387.

Coccidies nouvelles ou peu connues. Tablettes zoologiques, II, 1887, p. 5 .

Siedlecki (M.). - Reproduction sexuée et début de la sporulation chez la coccidie des tritons (Coccidium proprium Schneider). C.R. Soc. de Biol., L, 1898 , p. 663 .

Simond (P.-L.). - L'évolution des Sporozoaires du genre Coccidium. Ann. Inst. Pasteur, XI, 1897, p. 545.

Steinhaus (J.). - Cytophagus tritonis, eine in den Darmepithelzellen parasitisch lebende Coccidie. Centralbl. f. Bakt., Abt. I, Or., IX, 1891, p. 50.

WASIELEWSKI (T. von). - Studien und Mikrophotogramme zur Kenntnis der pathogenen Protozoen. I, Untersuchungen über den Bau, die Entwicklung und über der pathogene Bedeutung der Coccidien, Leipzig, 1904.

(A suivre).

Laboraloire de Zoologie el Parasitologie de la Facullé de médecine de Lille et Station aquicole Grimaldi à Saint-Jean de Losne (Còte-d'Or). 